\title{
Bestimmung der Diffusionskonstante von Leitungselektronen in Anthracen aus dem Impulsabfall bei Driftexperimenten
}

\author{
N. KARL* E. SCHMID * und M. SEEgER ** \\ Institut für Physikalische Chemie der Universität Freiburg \\ und Kristallabor des II. und III. Physikalischen Instituts der Universität Stuttgart
}

(Z. Naturforsch. 25 a, 382-391 [1970]; eingegangen am 18. Dezember 1969)

\begin{abstract}
Drift-current-pulses in semiconductors with vanishing dark conductivity measured according to the method of Kepler and LeBlanc deviate from the expected rectangular pulse shape even under ideal experimental conditions. In this work it will be shown that among other processes the diffusion of current carriers is responsible for the deviation. Under appropriate experimental conditions one is able to measure diffusion-constants in anthracene by a method to be described. Within experimental error the diffusion-constant obtained agrees with the value calculated from the Einsteinrelation using the independently measured drift-mobility. The differences in method from the known techniques used with dark conducting semiconductors are discussed. The measurements succeed only with very good crystals for which quality criteria are given.
\end{abstract}

\section{Einleitung, Überblick}

Unter idealen Bedingungen sollten LadungsträgerDriftstromimpulse nach der Technik von KEPLER und LeBLANC ${ }^{1}$ rechteckförmig sein. Es gibt jedoch viele Einflüsse, die zu Abweichungen führen ${ }^{2}$. Abweichungen mit physikalisch interessanten Ursachen erfolgen u. a. durch Diffusion und Rekombination ${ }^{2,3}$.

In der vorliegenden Arbeit wird theoretisch und experimentell gezeigt, daß es möglich ist, alle anderen Einflüsse so klein zu halten, daß aus der Abweichung von der idealen Driftstromkurve die Diffusionskonstante der Leitungselektronen in Anthracen-Einkristallen ermittelt werden kann. Bisher sind unseres Wissens in der Literatur keine solchen Untersuchungen bekannt geworden. Auch handelt es sich um die erste Bestimmung von Ladungsträgerdiffusionskonstanten in organischen Halbleitern. Die Methode erlaubt eine Aussage darüber, ob die Ladungsträger wirklich im Sinne des Bändermodells frei sind. Die Ergebnise an Anthracen bestätigen im Rahmen der Meßgenauigkeit die Einstein-Relation bzw. führen unter Voraussetzung der EinsteinRelation zu einer unabhängigen Bestimmung der Driftbeweglichkeit neben der üblichen Driftmethode ${ }^{1}$.

* Sonderdruckanforderungen an Dr. N. KARL, III. Physikalisches Institut der Universität Stuttgart, Kristallabor, D-7000 Stuttgart-1, Azenbergstraße 12.

** Jetzt: Institut für Makromolekulare Chemie der Universität Freiburg (Brg.), D.7800 Freiburg (Brg.), Stefan-MeierStraße 31.

\section{Theorie}

2.1. Nichtanwendbarkeit der klassischen Methoden zur Bestimmung der Diffusionskonstanten bei Halbleitern mit großer dielektrischer Relaxationszeit

HAYNES und SHOckley ${ }^{4}$ erzeugten in ihrem bekannten Experiment zur Bestimmung der Beweglichkeiten in einem Germaniumstab durch Injektion sprungartig eine höhere Konzentration an Ladungsträgern und "schwemmten" diese mit Hilfe eines Driftfeldes durch den Kristallstab. An einer entfernten Stelle konnte das Vorbeilaufen der Minoritätsträger und damit deren Verteilungsfunktion mit einer Kollektorsonde selektiv nachgewiesen werden. Die Verschmierung der Sprungfunktion führten die Autoren auf die Diffusion der Minoritätsträger zurück.

LAWRANCE und GIBSON ${ }^{5}$ erzeugten in einer ähnlichen Anordnung die zusätzlichen Ladungsträger durch Anregung mit einem kurzen Blitz als $\delta$-funktionsartige Verteilung und stellten am Kollektor Gauß-Funktionen mit zeitabhängigen $1 / e$-Wertsbreiten von $2 \cdot \sqrt{4 D t}$ (D:Diffusionskonstante) fest, wie dies die Diffusionstheorie fordert. Dobrovol'skII

1 R. G. Kepler, Phys. Rev. 119, 1226 [1960]. - O. H. Le BlanC JR., J. Chem. Phys. 33, 626 [1960].

2 N. KARL, Dissertation, Freiburg 1968.

3 G. Sommer, Diplomarbeit, Stuttgart 1969.

4 J. R. Haynes u. W. Shockley, Phys. Rev. 81, 835 [1951].

5 R. Lawrance u. A. F. Gibson, Proc. Phys. Soc. London 65 B, 994 [1952]. 
und Lyashenko ${ }^{6}$ arbeiteten ähnlich, jedoch ohne Driftfeld, s. a. ${ }^{7}$.

Bei diesen Messungen liegt stets der raumladungsfreie Fall vor, weil einmal erzeugte Überschußladungen wegen der kleinen dielektrischen Relaxationszeiten (die im wesentlichen eine Folge der relativ hohen Dunkelleitfähigkeiten sind) in $10^{-9}$ bis $10^{-12}$ sec kompensiert werden oder abfließen. Man hat stets nur ladungskompensierte Überschußkonzentrationen vorliegen und gewinnt auf einfache Weise Aussagen nur im nichteigenleitenden Fall und nur über Minoritätsträger *, ferner nur, wenn im Vergleich zu den schon vorhandenen Konzentrationen nur wenige zusätzliche Ladungsträger erzeugt wurden.

Bei Halbleitern mit geringer Dunkelleitfähigkeit und damit großer dielektrischer Relaxationszeit ist es schwierig, raumladungsfrei zu arbeiten. Im allgemeinen muß man die Coulomb-Wechselwirkung unter den Ladungsträgern in Kauf nehmen, die z. B. über die Abstoßung gleichnamiger Ladungsträger zu einer starken Verbreiterung der Verteilungen führt. Außerdem werden an den Elektroden Verschiebungsströme induziert. Beides erfordert eine wesentliche Modifikation der Versuchsanordnung und der theoretischen Beschreibung. Dafür können über die Erzeugung von beweglichen Raumladungen des einen oder des anderen Vorzeichens Aussagen über beide Vorzeichen gewonnen werden. Dies wird im folgenden gezeigt.

Wir betrachten eine Halbleiterkristallscheibe mit einer im Verhältnis zur Meßzeit großen dielektrischen Relaxationszeit. An diese kann im Dunkeln ein Feld angelegt werden, ohne daß sich der Kristall innerhalb der Meßzeit wesentlich polarisiert. Das interne Feld ist damit zunächst quellenfrei, also konstant (eindimensional beschreibbare Anordnung) und durch den Potentialabfall zwischen den Elektroden gegeben. Diese Verhältnisse werden nicht wesentlich gestört, wenn wir auf einer Seite des Kristalls momentan sehr wenige Elektron-Loch-Paare erzeugen. Je nach Feldrichtung wird das eine oder andere Vorzeichen mit konstanter Geschwindigkeit durch den Kristall driften. Die gesamte driftende, kleine Raumladung kann nach der Methode nach KePLER und LeBlanc ${ }^{1}$ in der durch die Elektroden

\footnotetext{
6 V. N. Dobrovol'skin u. V. I. Lyashenko, Pribory i Tekhnika Eksperim. 4, 118 [1961].

7 F. S. Goucher, Phys. Rev. 81, 475 [1951].
}

gebildeten Kondensatoranordnung (s. Abb. 1) als Verschiebungsstrom gemessen werden, der solange einen konstanten Wert beibehält, bis die $\delta$-funktionsartige Ladungsverteilung an der Gegenelektrode bei $L^{\prime}$ angekommen und entladen ist.

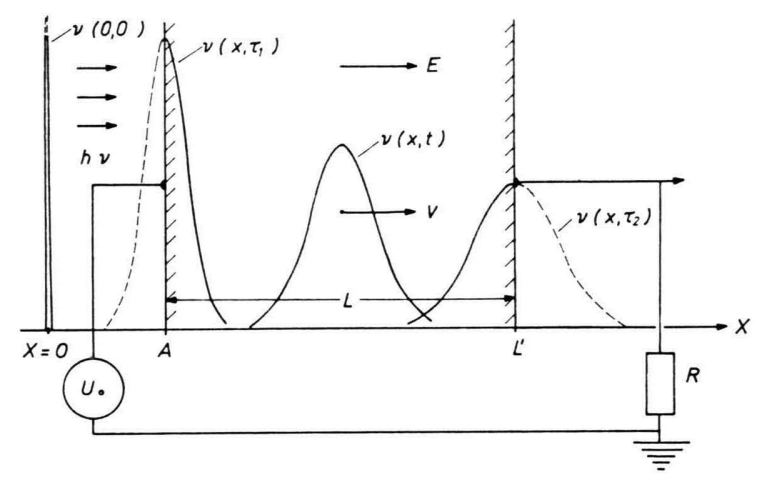

Abb. 1. Meßanordnung. Der Kristall erstreckt sich von $A$ bis $L^{\prime}$. Im Modell läuft eine endlich breite Gauß-Verteilung bei $A$ in den Kristall, die am fiktiven Punkt $x=0$ als $\delta$-Verteilung vorgelegen haben soll.

Ist die $\delta$-Funktion inzwischen breitgelaufen, jedoch noch schmal gegen die Kristalldicke $L$, so fließt im äußeren Stromkreis so lange ein konstanter Strom, so lange die Verteilung die Elektrode bei $L^{\prime}$ noch nicht erreicht hat. Werden die dort ankommenden Ladungen sofort kompensiert, d. h., hat man einen Ohm'schen Kontakt, so wird die Stromabnahme in einem kleinen Zeitintervall $\mathrm{d} t$ durch die Ladungsträger gegeben, die gerade in die Elektrode hineinlaufen; die Stromänderung $\mathrm{d} j / \mathrm{d} t$ wird $\mathrm{der}$ Verteilungsfunktion der Ladungsträger folgen und u. a. Information über eine Diffusionsverbreiterung beinhalten. Dieser Grundidee folgend wird im weiteren eine detaillierte Theorie abgeleitet.

\subsection{Bestimmung von Ladungsträgerverteilungs- funktionen durch Differentiation von Driftstrom- impulsen}

Eine Kristallscheibe der Dicke $L$, die in $y$ - und $z$-Richtung groß ist gegen $L$ und die Fläche $F$ hat, befinde sich zwischen $x=A$ und $x=L^{\prime}$ (Abb. 1). Im Kristall laufe eine Verteilung von Ladungsträgern der Konzentration $v(x, t)\left(\mathrm{cm}^{-1}\right)$ in einem elektrischen Feld $E$.

\footnotetext{
* Die Majoritätsträger folgen innerhalb der dielektrischen Relaxationszeit den Minoritätsträgern und sorgen für Raumladungsfreiheit, ohne sich in ihren spezifischen Eigenschaften bemerkbar zu machen.
} 
Der divergenzfreie Gesamtstrom setzt sich in jedem Volumelement aus Leitungsstrom $j_{\mathrm{L}}$, Diffusionsstrom $j_{\mathrm{D}}$ und Verschiebungsstrom $j_{\mathrm{V}}$ zusammen.

$$
\begin{aligned}
& j(x, t) \equiv j(t)=j_{\mathrm{L}}(x, t)+j_{\mathrm{D}}(x, t)+j_{\mathrm{V}}(x, t) \\
& =q \mu E(x, t) v(x, t)-q D \frac{\partial v(x, t)}{\partial x}+\varepsilon \varepsilon^{*} \frac{\partial E(x, t)}{\partial t} ;
\end{aligned}
$$

$q, \mu$ : Elementarladung, Beweglichkeit; für Löcher positiv, für Elektronen negativ zu nehmen; $\varepsilon, \varepsilon^{*}:$ relative und absolute Dielektrizitätskonstante.

Liegen Elektronen und Löcher vor, so ist jedes Glied getrennt anzuschreiben und zu addieren. In diesem Falle ist bei einer Überlappung der Verteilungsfunktionen Gl. (1) noch durch ein Rekombinationsglied zu ergänzen. Bei der gewählten Versuchsanordnung geht es jedoch nur in die Anfangsverteilung ein und kann im folgenden zunächst vernachlässigt werden.

Integration der Gl. (1) von $x=A$ bis $x=L^{\prime}$ ergibt mit der Bedingung einer zeitlich konstanten Potentialdifferenz zwischen den Elektroden (die man durch eine niederohmige Spannungsquelle $U_{0}$ und einen kleinen Widerstand $R$ mit $R j(t) F \ll U_{0}$ realisieren kann) und eines örtlich konstanten Feldes $E_{0}=U_{0} / L$ im Kristall (Raumladung der Verteilung $q \int_{A}^{L^{\prime}} v(x, t) \mathrm{d} x=q N$ pro $y-z$-Flächenelement sei so klein, daß sie nicht wesentlich zum Feld beiträgt, d. h. $\left.q N \ll \varepsilon \varepsilon^{*} E_{0}\right)$.

$$
j(t)=\frac{q \mu E_{0}}{L} \int_{A}^{L^{\prime}} \nu(x, t) \mathrm{d} x-\frac{q D}{L} \int_{\dot{A}}^{L^{\prime}} \mathrm{d} v(x, t) .
$$

Befindet sich die Verteilung ganz im Bereich zwischen $A$ und $L^{\prime}$, so macht sich Diffusion nicht im außen gemessenen Strom bemerkbar, weil dann $v(A)=v\left(L^{\prime}\right)=0$. Der Strom ist dann genau so groß, wie wenn eine homogene Trägerkonzentration $v_{0}=\frac{1}{L} \int_{A}^{r^{\prime}} v(x) \mathrm{d} x$ im ganzen Kristall vorläge.

Sei zunächst $D=0$. Eine $\delta$-funktionsartige Ladungsverteilung laufe mit der Geschwindigkeit $v=\mu E_{0}$ durch den Kristall. Dies führt zu einem konstanten Strom $j_{0}=q \mu E_{0} N / L$, der nach der Zeit $\tau_{2}-\tau_{1}=\tau=L / v$ plötzlich auf Null fällt, da die Ladungsträger die Gegenelektrode erreicht haben. Dies führt bekanntlich zu dem idealen, zeitlich rechteckförmigen Driftstromimpulsverlauf im äußeren Kreis, siehe Abb. 2 a. Die einzigen Informationen, die man daraus gewinnen kann, sind die erzeugte
Ladungsträgeranzahl $N F$ und die Beweglichkeit $\mu=v / E$.

Wir betrachten nun eine im Vergleich zur Kristalldicke stets schmale Verteilung $v\left(x, \tau_{1}\right)$, die bei $A$ in den Kristall läuft, ohne gleichzeitig ihre Gestalt zu ändern $(D=0)$. Wenn sie gerade ganz im Kristall ist, lassen wir Verbreiterungsmechanismen, etwa als Beispiel die Diffusionsverbreiterung, zu $(D \neq 0)$, die solange wirken sollen, bis die Verteilung in der Gestalt $v\left(x, \tau_{2}\right)$ an der Gegenelektrode ankommt. Während des Hinweglaufens über diese Elektrode sei wiederum $D=0$. Unter diesen Umständen können wir uns auf das l. Glied der Gl. (2) beschränken, das den reinen Driftvorgang beschreibt.

Anschaulich ist nun klar, daß wegen der endlichen Zeit, die beim Ein- und Austreten der Verteilung verstreicht, die Ecken der idealen Driftstromkurve gerundet werden, siehe Abb. $2 \mathrm{~b}$.

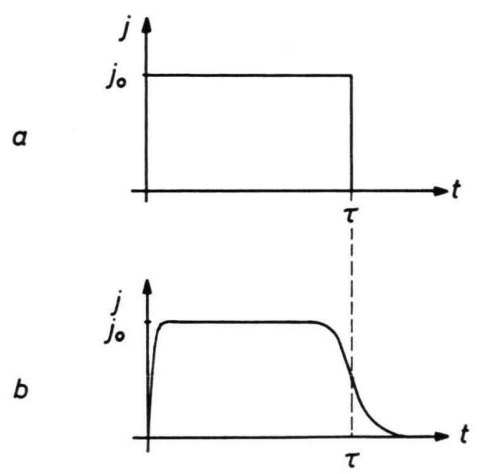

Abb. 2 a. Idealer Driftstromimpuls für eine sich nicht verändernde $\delta$-Verteilung; $2 \mathrm{~b}$ : realer Driftstromimpuls, wie er etwa beim Vorliegen der Verteilung der Abb. 1 gemessen wird.

Zum einfacheren Verständnis führen wir nun ein mit der Verteilung mitbewegtes Koordinatensystem $x^{\prime}$ ein, das seinen Ursprung an einer mathematisch geeigneten Stelle der Verteilungsfunktion hat. Zunächst seien im Gedankenexperiment beide Elektroden so weit von $x^{\prime}=0$ entfernt, $\mathrm{da} \beta$ sich die Vertejlung ganz außerhalb des Kristalls befindet (Abb). 3 a). Nun lassen wir die Elektroden mit der Relativgeschwindigkeit $v_{0}=\left|\mu E_{0}\right|$ über die Verteilung (im Bild nach links) hinweglaufen. Nur der Wert des Integrals $\int v\left(x^{\prime}, t\right) \mathrm{d} x^{\prime}$ im Gebiet zwischen den Elektroden trägt zum gemessenen Strom bei:

$$
j(t)=\frac{q \mu E_{0}}{L}\left(\int_{A-v_{0} t}^{\infty} v\left(x^{\prime}, \tau_{1}\right) \mathrm{d} x^{\prime}-\int_{L^{\prime}-v_{0} t}^{\infty} v\left(x^{\prime}, \tau_{2}\right) \mathrm{d} x^{\prime}\right),
$$

wobei der Strom konstant ist, wenn sich die Vertei- 


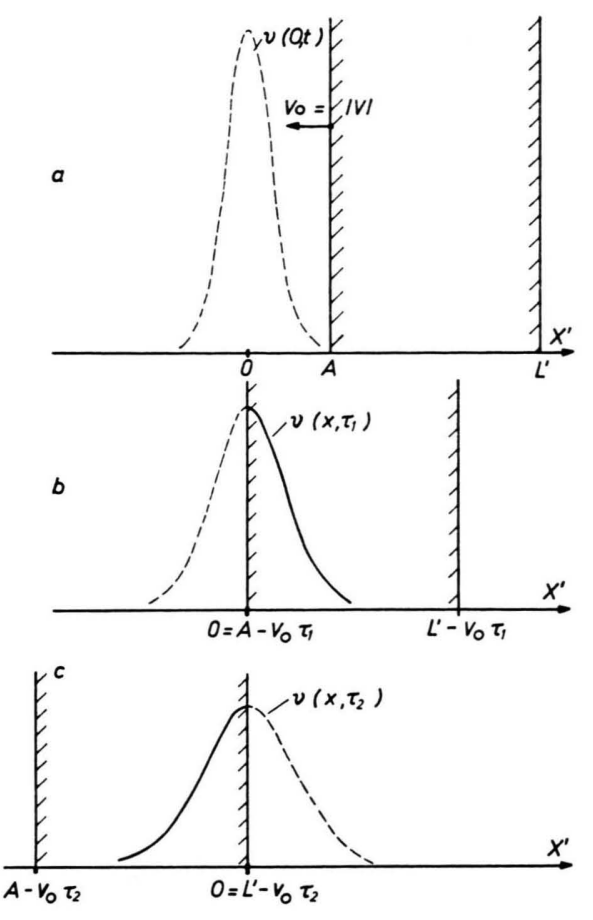

Abb. 3. Mit der Verteilung mitbewegtes Koordinatensystem.

lung ganz im Gebiet zwischen den Elektroden befin$\operatorname{det}$ (s. o.).

Differentiation ergibt:

$\frac{\mathrm{d} j(t)}{\mathrm{d} t}=\frac{q \mu E_{0} v_{0}}{L}\left\{v\left(A-v_{0} t ; \tau_{1}\right)-v\left(L^{\prime}-v_{0} t ; \tau_{2}\right)\right\}$.

Nehmen wir nun noch die Rücktransformation auf die örtliche Breite der Verteilung beim Durchqueren der jeweiligen Elektroden vor:

$$
x^{\prime}+A=v_{0} t \quad \text { bzw. } \quad x^{\prime}+L^{\prime}=v_{0} t,
$$

so wird klar, daß die Differentiation des Stromes nach der Zeit die Gestalt der Verteilungsfunktion beim Durchlaufen der Elektrode $A$ bzw. $L^{\prime}$ liefert (Abb. 3 b, c) :

$\left(\frac{\mathrm{d} j}{\mathrm{~d} t}\right)_{A}=\beta v\left(-x^{\prime}, \tau_{1}\right) \quad$ mit $\quad \beta=\frac{q \mu E_{0} v_{0}}{L}=\frac{q v_{0}{ }^{2}}{L}$ $\left(\frac{\mathrm{d} j}{\mathrm{~d} t}\right)_{L^{\prime}}=-\beta v\left(-x^{\prime}, \tau_{2}\right)$,

d. h., die Verteilung wird beim Durchqueren der Elektroden in negativer Richtung "abgetastet“. Sie erscheint gespiegelt, was auch aus der Anschauung unmittelbar folgt. Läuft eine 2. Verteilung mit umgekehrtem Ladungsvorzeichen in entgegengesetzte Richtung, so überlagert sich dieser Effekt linear additiv (Rekombination vernachlässigt) .

\subsection{Diffusionsverbreiterung}

Bekanntlich diffundiert eine $\delta$-Verteilung in eine Gauß-Verteilung der Gestalt

$$
v\left(x^{\prime}, t\right)=\frac{N}{2 \sqrt{\pi D t}} \exp \left\{-x^{\prime 2} / 4 D t\right\}
$$

breit, siehe z. B. ${ }^{8}$. Es wäre also am günstigsten, an der Stelle $A$ eine $\delta$-Verteilung zu erzeugen. Da im später beschriebenen Experiment die Erzeugung von Ladungsträgern bei $A$ mit stark absorbiertem Licht eines kurzen Blitzes erfolgt (lichtdurchlässige Elektrode), ist die Anfangsverteilung jedoch durch die während der endlich langen Blitzdauer von den ersten Ladungsträgern im Feld zurückgelegte Strecke und durch die Eindringtiefe des Lichts ${ }^{*}$, also durch den reziproken Absorptionskoeffizienten bestimmt. Wir tragen dem Rechnung dadurch, daß schon bei $A$ eine Gauß-Verteilung angenommen wird, die man sich aus einer $\delta$-Verteilung bei $x=0$ (Abb. 1) entstanden denken kann, d. h., man muß nur den Zeitnullpunkt der Gl. (6) zurückverlegen. Die zusätzlich während der Durchquerung des Kristalls erfolgte Diffusionsverbreiterung der $1 / e$-Wertsbreite beträgt nur:

$$
\Delta x_{2}-\Delta x_{1}=2 \sqrt{4 D \tau_{2}}-2 \sqrt{4 D \tau_{1}} .
$$

Da man die Breite der Anfangs- und Endverteilung im Kristall nach Gl. (5) messen kann, läßt sich mit Gl. (7) die Diffusionskonstante bestimmen. Gleichung (7) läßt sich in folgende Form bringen $\left(\Delta x_{2}=2 \sqrt{4 D \tau_{2}}\right)$ :

$$
\left(\Delta x_{2}\right)^{2}-\left(\Delta x_{1}\right)^{2}=16 D \tau \quad \text { mit } \quad \tau=\tau_{2}-\tau_{1},
$$

deren linke Seite gegen verschiedene Transitzeiten $\tau$ (durch Variation des Driftfeldes) aufgetragen eine Gerade mit der Steigung $16 \mathrm{D}$ ergeben sollte. Man erwartet keine Gerade, wenn nicht das $\sqrt{t}$-Gesetz der Gl. (7) vorliegt, d. h., wenn Verbreiterungen mit anderen Mechanismen, etwa $\sim t$ stattgefunden haben.

\subsection{Störeinflüsse}

Unter Störeinflüssen seien solche verstanden, die ebenfalls zu einer (echten oder scheinbaren) Verbreiterung der Verteilung führen:

\footnotetext{
8 W. Jost, „Diffusion“, Darmstadt 1957.

** Der Beitrag zur Anfangsverteilung, der von der endlichen Eindringtiefe des Anregungslichts herrührt, wird bei der Bestimmung der Verteilungsfunktion durch Differentiation nicht mit erfaßt; er ist im allgemeinen klein und kann vernachlässigt werden.
} 
a) Unebenheit oder Keilförmigkeit des Kristalls,

b) Wechselwirkung der Ladungsträger mit flachen Haftstellen (Kinetik),

c) Coulomb-Wechselwirkung mit geladenen Haftstellen,

d) Coulomb-Wechselwirkungen unter den freien Ladungsträgern.

Über weitere Einflüsse siehe ${ }^{2,9,10}$.

Zu a): Eine Streuung der Dicke $\pm \Delta L$ über die Fläche des Kristalls führt zu einer Laufzeitstreuung $\tau \pm \Delta \tau=(L \pm \Delta L)^{2} / \mu U_{0}$, d. h. $\Delta \tau \sim 2 \Delta L$. Die Zwei rührt daher, daß z. B. bei größerem $L$ die durchlaufene Strecke größer und gleichzeitig das Feld und damit die Geschwindigkeit kleiner ist. Die Planparallelität der Kristallscheiben muß daher sehr großen Anforderungen genügen.

Zu b): Die Wechselwirkung mit flachen Haftstellen führt dazu, daß Ladungsträger, die kurz von einer Haftstelle festgehalten wurden, der Verteilung nachhinken. Wenn die Anzahl solcher Wechselwir. kungsprozesse auf dem Weg eines Ladungsträgers durch den Kristall klein ist, so kommen viele Ladungsträger ohne Wechselwirkung an und nur wenige verspätet. Die Verteilung wird asymmetrisch und erhält einen flachen Ausläuser. Durch Auswertung der Halbwertsbreite statt der $1 / e$-Wertsbreite kann man diesen Fehler mitunter ausschalten.

Liegen viele Wechselwirkungsprozesse mit flachen Haftstellen vor, so kann sich die mittlere Transitzeit auf ein Mehrfaches des Wertes $\tau_{0}$ ohne Haftstellen verlängern. Nennen wir die längere Zeit $\tau_{\text {eff }}$. Die zugehörige statistisch verbreiterte Verteilungsfunktion kann in Analogie zum Durchgang einer Substanz durch eine Gaschromatographen-Absorptionssäule abgeleitet werden ${ }^{2}$ und liefert für eine $\delta$-förmige Ausgangsverteilung eine Gauß-Verteilung, die nichts mit Diffusion zu tun hat. Ihre zeitliche Breite beträgt nun

$$
\Delta t_{1 / e}=\sqrt{8 \tau_{\text {eff }} \cdot \tau_{\mathrm{B}} / \tau_{0}},
$$

wobei $\tau_{\mathrm{B}}$ die Zeit ist, die sich ein Ladungsträger im Mittel zwischen zwei Einfangprozessen frei im Band bewegen kann. Diese Verbreiterung ist wesentlich. Sie wurde von PAPADAKIS ${ }^{11}$ vernachlässigt.

Zu c): Trägt der Kristall stationäre Raumladungen, die etwa von vorausgehenden Experimenten in tiefen Haftstellen sitzen, so wird das Feld im Kristall nicht mehr quellenfrei und damit inhomogen; dies führt prinzipiell zu einer Verlängerung der

9 M. SEEGER, Diplomarbeit, Freiburg 1968.

10 E. SChmid, Diplomarbeit, Stuttgart 1969.
Transitzeit ${ }^{2}$. Die Ladungsträger laufen ein Stück im Kristall langsamer und holen die verlorene Zeit im schnelleren Teil nicht wieder ein. (Die Transitzeit ist umgekehrt proportional zur Geschwindigkeit; deren Integral $\int_{A}^{L^{\prime}} v \mathrm{~d} x$, wegen $v \sim E$ und $\int_{A}^{L^{\prime}} E \mathrm{~d} x=U_{0}$, bleibt unverändert.)

Ist die Raumladung in $y-z$-Richtung homogen verteilt, so ergeben sich keine örtlichen Streuungen der verlängerten Transitzeit und damit keine scheinbaren Verbreiterungen durch eine Auffächerung der Laufzeiten.

Die stationäre Raumladung $q \cdot \tilde{v}$ jedoch, die sich gerade unter der Verteilungsfunktion befindet, führt zu einem Feldstärkeunterschied

$$
E_{2}-E_{1}=\frac{q}{\varepsilon \varepsilon^{*}} \int_{x_{1}}^{x_{2}} \tilde{\nu} \mathrm{d} x
$$

zwischen der Front der Verteilung bei $x_{2}$ und der rückwärtigen Flanke bei $x_{1}$ und wirkt dadurch verbreiternd, wenn die stationäre Raumladung dasselbe Vorzeichen hat wie die driftenden Ladungen.

Damit unterscheidet sich der Effekt - bis auf die mögliche Ortsabhängigkeit des verbreiternden Feldes - nicht von der Coulomb-Abstoßung unter den freien Ladungsträgern. Diese wird unter d) behandelt. Hat die stationäre Raumladung das umgekehrte Vorzeichen, so findet eine Verschmälerung der driftenden Verteilung statt.

Zu d): Wenn die Anzahl der Ladungsträger $N$ in der Verteilung nicht vernachlässigbar klein ist, z. B. nach starker Anregung, so führt auch die CoulombAbstoßung unter den freien Ladungsträgern in der Verteilung zu einer Verbreiterung, da nun ein Feldstärkeunterschied

$$
E_{2}-E_{1}=\frac{q}{\varepsilon \varepsilon^{*}} \int_{x_{1}}^{x_{2}} v(x) \mathrm{d} x
$$

zwischen den Flanken der Verteilung herrscht. Einfach analytisch behandeln läßt sich in der gewählten eindimensional beschreibbaren Anordnung nur eine Rechteckverteilung, mit der wir nun unsere tatsächlich vorliegende Verteilung annähern wollen. Eine Rechteckverteilung der Ausgangsbreite $\Delta x\left(\tau_{1}\right)$ und der Ausgangskonzentration $v_{0}$ verbreitert sich linear mit der Zeit ${ }^{2,9,11}$.

11 A. C. Papadakis, J. Phys. Chem. Solids 28, 641 [1967]. 


$$
\Delta x(t)=\Delta x\left(\tau_{1}\right)\left[1+\left(t-\tau_{1}\right) \frac{\mu q v_{0}}{\varepsilon \varepsilon^{*}}\right] .
$$

Dies rührt daher, daß eine homogene Raumladung bei Abwesenheit von Ladungsträgern des anderen Vorzeichens hyperbolisch mit der Zeit mit einer konzentrationsabhängigen Relaxationszeit

$$
\tau_{1 / 2}=\varepsilon \varepsilon^{*} / \mu q v_{0}
$$

abfließt * und daß sich daran durch Beschneidung zu einer Rechteckverteilung nichts ändert. Die Forderung der Erhaltung der Gesamtladung führt zu Gl. (10) .

Die Coulomb-Verbreiterung kann gemäß Gl. (10) klein gehalten werden, wenn die Anzahl der Ladungsträger $N=v_{0} \Delta x\left(\tau_{1}\right)$ in der Verteilung nicht zu groß gemacht wird. Dabei ändert sich an der Diffusionsverbreiterung nichts, da sie gemäß Gl. (6) und (7) unabhängig vom Amplitudenfaktor der Gauß-Verteilung ist. Durch sehr schwache Anregung kann somit die Coulomb-Verbreiterung klein gegen die Diffusionsverbreiterung gemacht werden. Experimentell bedingt dies jedoch die Messung sehr kleiner Impulsströme.

Verlangen wir z. B., daß eine gemessene Breite von $\Delta x\left(\tau_{\mathbf{2}}\right)=1,1 \xi_{0}$ höchstens zu $10 \%$ von der Coulomb-Wechselwirkung unter den Ladungsträgern bedingt sein soll und da $\beta$ die Breite $1,0 \xi_{0}$ von der Diffusion herrühren soll, d. h.

$$
0,1 \xi_{0}=\left\{\Delta x\left(\tau_{2}\right)-\Delta x\left(\tau_{1}\right)\right\}_{\text {Coul. }}=\frac{\mu q N \tau}{\varepsilon \varepsilon^{*}}
$$

[s. Gl. (10)] und $\xi_{0}=2 \sqrt{4 D \tau}$ [s. Gl. (8)], so folgt mit $\alpha=\xi_{0} / L$ (relative Verbreiterung im Verhältnis zur Kristalldicke)

$$
10 q \mu N \tau / \varepsilon \varepsilon^{*} \leqq 2 \sqrt{4 D \tau}
$$

oder, anders geschrieben, für die höchstens zulässige Ladung in der Verteilung

$$
q N \leqq \frac{0,8 \varepsilon \varepsilon^{*}}{\alpha L} \cdot \frac{k T}{q} .
$$

Hierbei wurde die Einstein-Relation $D=\mu k T / q$ für diese Abschätzung als gültig angenommen ( $k$ : Boltzmann-Konstante, $T$ : absolute Temperatur).

Für den Strom findet man

$$
j=q N / \tau \leqq 12,8 \mu \varepsilon \varepsilon^{*}(1 / \alpha L)^{3}(k T / q)^{2} .
$$

\footnotetext{
* Dies folgt einfach aus Strom-, Kontinuitäts- und PoissonGleichung unter der Voraussetzung, daß die Leitfähigkeit ausschließlich auf die bewegliche Raumladung zurückzuführen ist.
}

Die Spannung, bei der gerade die geforderte Diffusionsverbreiterung auftritt, errechnet sich zu $U_{0}=\frac{L^{2}}{\mu \tau}=\frac{16}{\alpha^{2}} \cdot \frac{k T}{q} \approx \frac{0,4}{\alpha^{2}}$ Volt (Zimmer-Temp.).

Man sieht, daß man mit sehr kleinen Spannungen arbeiten muß.

$L=0,01 \mathrm{~cm}$

\begin{tabular}{cccr}
\hline \multicolumn{1}{c}{$\alpha$} & $j\left[\mathrm{~A} / \mathrm{cm}^{2}\right]$ & $\tau[\mathrm{sec}]$ & $U_{0}[\mathrm{~V}]$ \\
\hline 0,033 & $6,9 \cdot 10^{-5}$ & $2,5 \cdot 10^{-7}$ & 400 \\
0,10 & $2,5 \cdot 10^{-6}$ & $2,5 \cdot 10^{-6}$ & 40 \\
0,33 & $6,9 \cdot 10^{-8}$ & $2,5 \cdot 10^{-5}$ & 4 \\
\hline$L=0,02 \mathrm{~cm}$ & & & \\
\hline 0,033 & $8,6 \cdot 10^{-6}$ & $1 \cdot 10^{-6}$ & 400 \\
0,10 & $3,1 \cdot 10^{-7}$ & $1 \cdot 10^{-5}$ & 40 \\
0,33 & $8,6 \cdot 10^{-9}$ & $1 \cdot 10^{-4}$ & 4 \\
\hline$L=0,03 \mathrm{~cm}$ & & & \\
\hline 0,033 & $2,5 \cdot 10^{-6}$ & $2,2 \cdot 10^{-6}$ & 400 \\
$0,10 *$ & $9,2 \cdot 10^{-8}$ & $2,2 \cdot 10^{-5}$ & 40 \\
$0,33 *$ & $2,5 \cdot 10^{-9}$ & $2,2 \cdot 10^{-4}$ & 4 \\
\hline$L=0,1 \mathrm{~cm}$ & & & 40 \\
\hline 0,033 & $6,9 \cdot 10^{-8}$ & $2,5 \cdot 10^{-5}$ & 400 \\
0,10 & $2,5 \cdot 10^{-9}$ & $2,5 \cdot 10^{-4}$ & 4 \\
0,33 & $6,9 \cdot 10^{-11}$ & $2,5 \cdot 10^{-3}$ & \\
\hline
\end{tabular}

Tab. 1 .

In Tab. 1 sind zur Orientierung einige Werte zusammengestellt (für $\mu=1 \mathrm{~cm}^{2} / \mathrm{V} \mathrm{sec}, \varepsilon=3,4$ ).

Praktisch lassen sich nur wenige der aufgeführten Beispiele realisieren, da man, durch die Dauer der Anregung und durch die Eindringtiefe des Lichts bedingt, stets eine gewisse Anfangsbreite der Verteilung vorliegen hat und sinnvollerweise eine dagegen große Verbreiterung messen sollte. Man muß also zu großen Transitzeiten $\tau$ und Kristalldicken $L$ übergehen, ist jedoch auf der anderen Seite durch das Rauschen der Meßapparatur auf gewisse nicht unterschreitbare Mindestströme angewiesen, was umgekehrt wegen des Faktors $1 / L$ in $\mathrm{Gl}$. (2) die Dicke beschränkt. Die angekreuzten Werte der Tab. 1 erweisen sich praktisch als günstig.

\section{Kristalle und Meßtechnik}

\subsection{Kristallherstellung, -Bearbeitung und-Prüfung}

Die Messungen wurden an Anthracen-Einkristallen durchgeführt, welche aus sorgfältig zonengerei- 
nigtem Anthracen („für Szintillation“ der Fa. Fluka) im Vakuum aus der Schmelze, nach Bridgman, gezogen worden waren.

Für die verwendeten Kristalle trafen folgende Qualitätsmerkmale zu:

1. Einheitliche Auslöschung unter dem Polarisationsmikroskop (orthoskopisch, kleine Apertur).

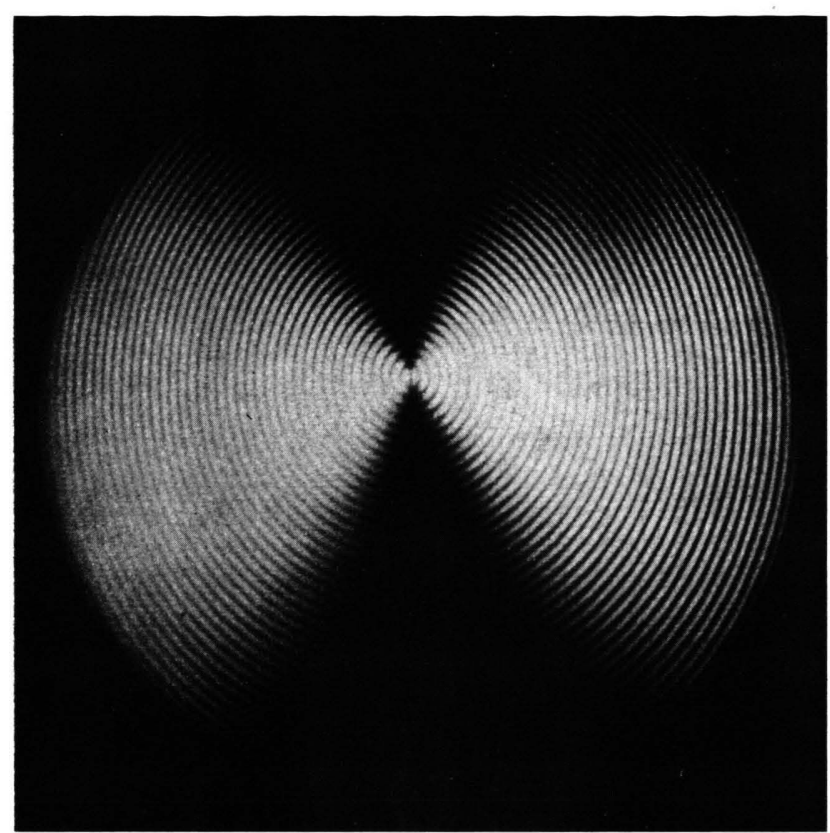

Abb. 4. Interferenzbild eines guten Anthracenkristalls von ca. $0,3 \mathrm{~mm}$ Dicke im monochromatischen, konvergenten polarisierten Licht; der Kristall lag mit der $a-b$-Ebene auf; eine optische Achse ist mit einem Universaldrehtisch senkrecht gestellt (Objektiv: Leitz UT 10; Bertrand-Linse).

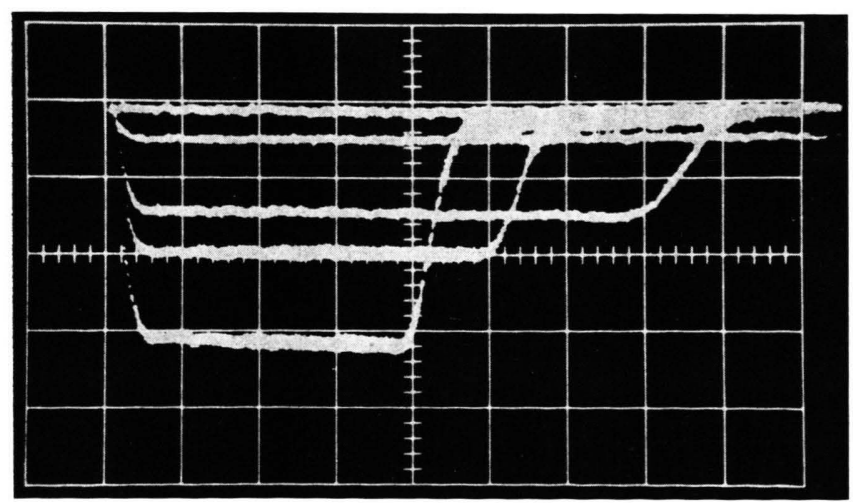

Abb. 5. Elektronendriftstromimpuls in einem 1,01 mm dicken Anthracenkristall bei (v. o. n. u.) 0, 150, 250, 350 und $450 \mathrm{~V}$ Driftspannung. Abszisse: $5 \mu$ sec pro Karo; Ordinate: 1,1 $\cdot 10^{-6} \mathrm{~A} / \mathrm{cm}^{2}$ pro Karo. Halbwertszeit des Blitzes hier ca. $1 \mu$ sec.

12 D. C. Hoesterey u. G. M. Letson, J. Phys. Chem. Solids 24, 1609 [1963].
2. Scharfe Interferenzfiguren gemäß Abb. 4 in monochromatischem Licht (Na-Dampf-Lampe) bei konoskopischer Betrachtung unter dem Polarisationsmikroskop.

3. Konzentration tiefer Haftstellen vernachlässigbar, s. Abb. 5: man beobachtet während der Meßdauer bei Driftstrommessungen keine Stromabnahme durch Haftstelleneinfänge. Lebensdauern der Ladungsträger ca. 1 msec.

4. Bei Zimmertemperatur wirksame flache Haftstellen für Elektronen durch Messung der Temperaturabhängigkeit der Transitzeit auszuschließen ${ }^{12}$.

5. Lebensdauer von Triplett-Excitonen $24 \mathrm{msec}$.

Zur Messung kamen Kristallscheiben von einigen Zehntel Millimetern Dicke und 0,1 bis $1 \mathrm{~cm}^{2}$ Fläche, die mit einer Fadensäge mit Xylol p. A. abgesägt und auf einer speziellen Poliermaschine ${ }^{13}$ auf Zellulose-Linsenputztuch der Fa. J. Green (vertreten durch Schoeps, Duisburg-Beek) mit Xylol eben poliert wurden. Mit einem Handapparat, bei dem der Kristall mit einer Mikrometerschraube in Schritten von ca. $1 \mu \mathrm{m}$ parallel vorgeschobenen werden konnte, ließen sich in einem letzten Arbeitsgang auf einer ebenen Glasplatte (mit Linsenputztuch und Xylol) die Unebenheiten auf einige $\mu \mathrm{m}$ reduzieren ${ }^{10}$. Diese Arbeitsgänge erfordern wegen der geringen Härte und wegen der leichten Spaltbarkeit des Anthracens viel Erfahrung, Fingerspitzengefühl und Geduld.

Die Ebenheit wurde schließlich nach kurzem Ätzen in Xylol interferometrisch geprüft. Abb. 6 a zeigt die Aufnahme eines guten Kristalls, Abb. 6 b die eines Kristalls mit gewölbter Oberfläche vor der Bearbeitung mit dem Handpoliergerät; (die Interferenzlinien lassen sich wie Höhenlinien deuten). Diese Interferogramme kommen im parallelen, monochromatischen Licht durch Interferenz der an der Vorderseite und durch den Kristall hindurch an der Rückseite reflektierten Welle zustande. Zu ihrer Herstellung genügt ein Auflichtmikroskop bei geeigneter Kondensoreinstellung (Beleuchtungs-Aperturblende fast zu).

Auf die Kristalle wurden im Vakuum Silberschichten aufgedampft ${ }^{2,10}$ mit einer Transparenz von ca. $50 \%$ und Flächenwiderständen von ca. $50 \Omega$ $\mathrm{cm}$. Die kristallographische Orientierung wurde nach den Messungen an Spaltstücken unter dem Polarisationsmikroskop nach den Daten von NAKADA ${ }^{14}$ festgestellt.

13 H. Rohrbacher, Diplomarbeit, Stuttgart 1969.

14 I. NAKAdA, J. Phys. Soc. Japan 17, 113 [1962]. 
N. Karl, E. Schmid und M. Segger, Bestimmung der Diffusionskonstante von Leitungselektronen in Anthracen aus dem Impulsabfall bei Driftexperimenten (S. 382).
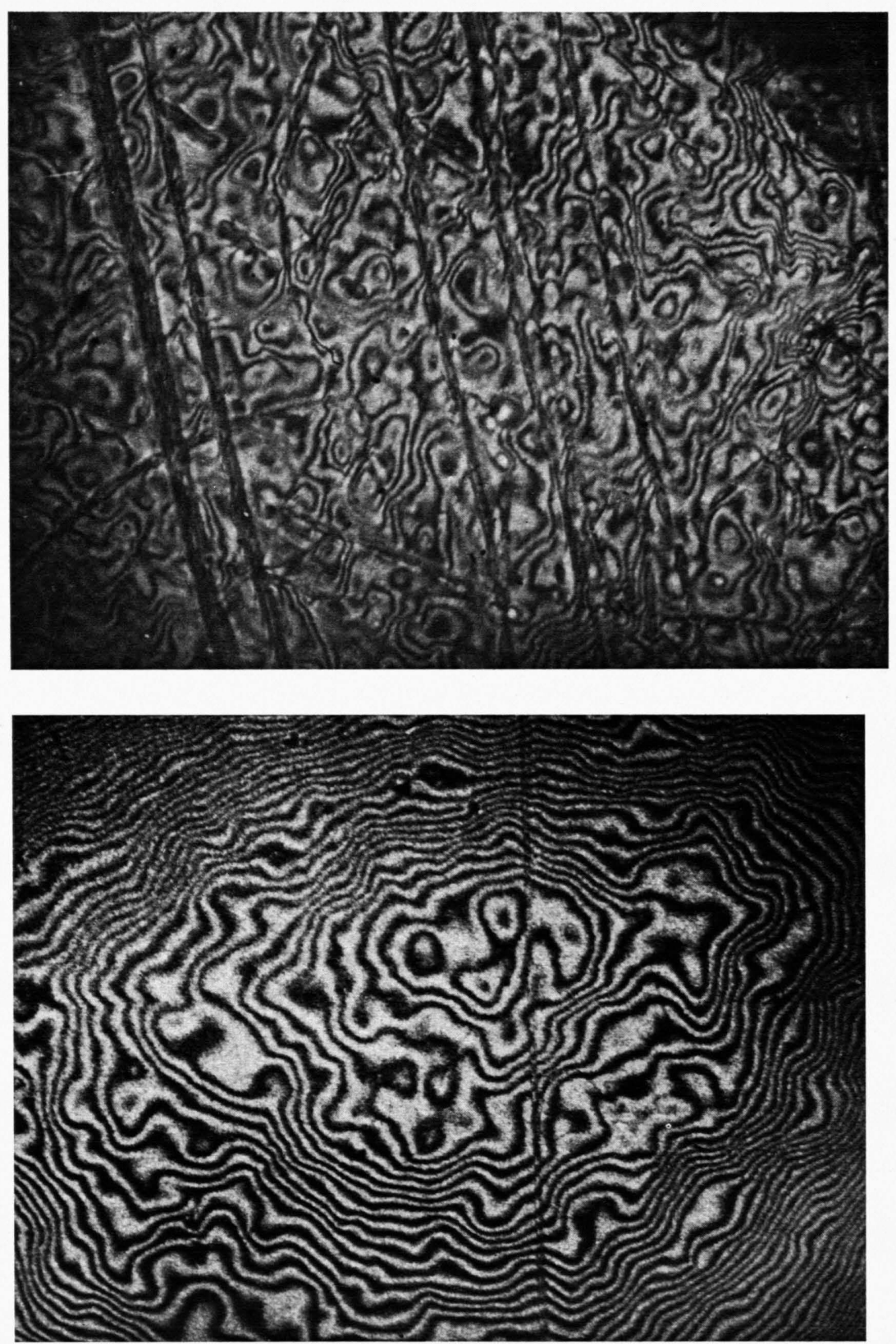

Abb. 6. Interferenzebenheitsprüfung von Anthracenkristallen; 6 a: nach, 6 b: vor der Endpolitur; polarisiertes Na-Licht; Fläche: 6 a: $12 \mathrm{~mm}^{2} ; 6 \mathrm{~b}: 4 \mathrm{~mm}^{2}$. 



\subsection{Optik}

Das Anregungslicht wurde durch Entladung eines $0,1 \mu \mathrm{F}$-Scheibenkondensators mit Zentralloch der Fa. Tobe Deutschmann, USA, über eine Funkenstrecke erzeugt. Der aperiodische Grenzfall konnte mit einem kurzen Stück Widerstandsdraht von etwa $0,3 \Omega$ im Entladekreis eingestellt werden. Die Halbwertsbreite des Blitzes betrug damit ca. 200 nsec. Die Entladung wurde durch ein Loch von $1 \mathrm{~mm} \phi$ und $1 \mathrm{~mm}$ Länge in einer Aluminiumoxyd-Keramikscheibe gegen eine ebenfalls durchbohrte anliegende Elektrodenplatte geführt und konnte dadurch sehr gut optisch abgebildet werden. Die Konstanz der Lichtintensität bei freiem ungetriggertem Ǔberschlag ließ sich durch Stickstoffspülung erhöhen.

Ladungsträger wurden im Anthracen im Gebiet der Volumerzeugung bei $250 \mathrm{~nm}$ (Interferenz-Reflexionsfilter der Fa. Schott) angeregt. Mit Graufiltern auf Quarzglas konnte die Lichtintensität abgeschwächt werden.

\subsection{Elektronik}

Die Messungen der Driftströme erfolgten über den Spannungsabfall, den sie an $R$ (Abb. 1) erzeugten, mit Hilfe eines Kathodenfolger-Tastkopfes (Tektronix P $170 \mathrm{CF}$ ), eines empfindlichen Vorverstärkers (Tektronix 1121) und eines Speicheroszillographen (Tektronix 549 mit Einschub 1 A 1). Die Eingangszeitkonstante, welche die Bandbreite bestimmt, wurde durch geeignete Wahl des Widerstandes $R$ klein gegen die Abfallzeiten der Driftstromimpulse gemacht und betrug mit typisch $10 \mathrm{k} \Omega$ ca. $0,5 \mu$ sec. Durch die Natur des Speicheroszillographen bedingt, sind schnelle Impuls-Anstiege oft nicht gleichzeitig mit langsamen Abfällen abbildbar. Unserer Konvention entsprechend sind Elektronenimpulse negativ aufgezeichnet.

Die Differentiation der Driftströme kann elektronisch durchgeführt werden, siehe ${ }^{2}$; da man die Ströme jedoch sehr klein halten muß, um Coulomb-Verbreiterung auszuschalten, werden sie sehr verrauscht. Deshalb erhielt graphische Differentiation den Vorzug.

Zur Messung befanden sich die Kristalle in einer elektrisch abschirmenden Metallmeßkammer in einem Vakuum von $10^{-4}$ Torr. Stets wurde mit kleinsten Spannungen und Lichtintensitäten begonnen. Zwischen den Einzelimpulsmessungen wurde der jeweilige Kristall mit mehreren Blitzen ,ausgeleuchtet“. So konnten Raumladungseffekte vermieden werden.

\section{Meßergebnisse}

Die Abb. 7 zeigt einen typischen Driftstromimpuls mit diffusionsverbreitertem Abfall. Bei Verlängerung der Transitzeit durch Verkleinerung der Spannung wurde die relative Breite $\alpha$ (s. Theorie) des Abfalls und damit der Verteilungsfunktion der ankommenden Ladungen größer. In Abb. 8 sind differenzierte Abfälle für drei verschiedene Transitzeiten (am sel-

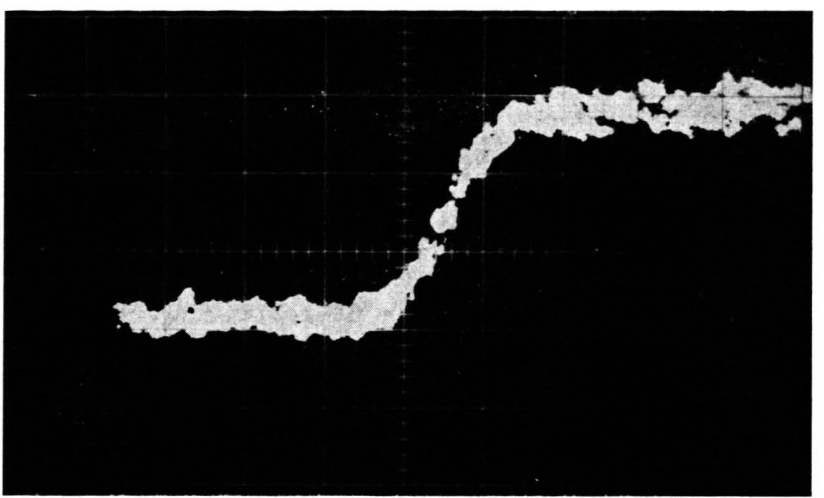

Abb. 7. Elektronen-Driftstromimpuls mit diffusionsverbreitertem Abfall; Anthracenkristall in $a$-Richtung $\pm 3^{\circ}$; Dicke: $280 \pm 3 \mu \mathrm{m}$; Fläche: $22 \mathrm{~mm}^{2}$; Spannung: $10 \overline{\mathrm{V}}$; Abszisse: $10 \mu$ sec pro Karo; Ordinate: $4 \cdot 10^{-8} \mathrm{~A} / \mathrm{cm}^{2}$ pro Karo; Lichtintensität : $10 \%$; drei Aufnahmen übereinander.

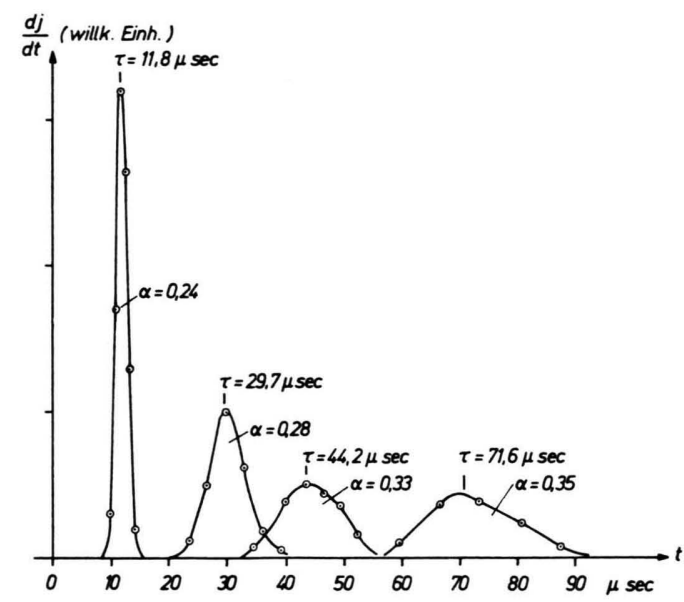

Abb. 8. Vier differenzierte Abfälle von Driftstromimpulsen am Kristall der Abb. 7 nach verschiedenen Transitzeiten. Die relative Breite (bezogen auf die Kristalldicke) nimmt mit der Zeit zu (Anfangsbreite nicht abgezogen!).

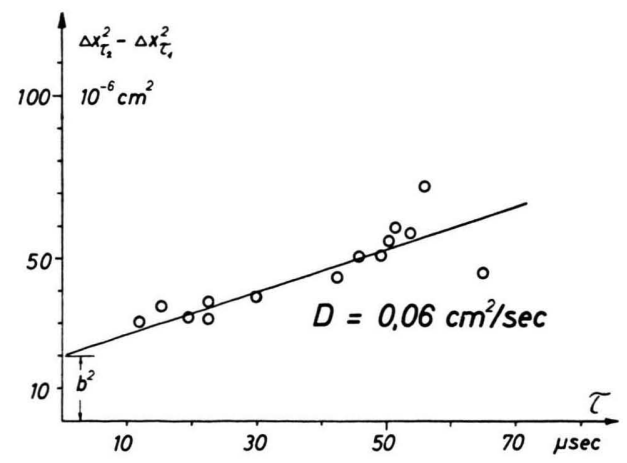

Abb. 9. Verbreiterungen vom Typ der Abb. 8, aufgetragen gegen die Transitzeit; die Steigung der Geraden ergibt die Diffusionskonstante. 
ben Kristall) dargestellt. Wertet man diese gemäß Gl. (8) aus, so findet man die Punkte der Abb. 9 (eingezeichnet sind Halbwertsbreiten, nicht $1 / e$ Wertsbreiten). Sie lassen sich im Rahmen der experimentellen Unsicherheit, die vor allem dadurch hereinkommt, daß die Meßkurven sehr verrauscht sind, durch eine Gerade beschreiben, aus deren Steigung eine Elektronendiffusionskonstante von $D=$ $0,06 \mathrm{~cm}^{2} / \mathrm{sec}$ (in $a$-Richtung) folgt. Die Beweglichkeit der Elektronen in dieser Kristallrichtung wurde $\mathrm{zu} \mu=1,75 \mathrm{~cm}^{2} / \mathrm{V}$ sec gemessen. Die hieraus über die Einstein-Relation errechnete Diffusionskonstante beträgt $D_{\text {theor. }}=0,044 \mathrm{~cm}^{2} / \mathrm{sec}$. Innerhalb der Fehlergrenze darf dies als Übereinstimmung gewertet werden.

In Abb. 9 fällt auf, daß auch für $\tau \rightarrow 0$ noch eine gewisse Breite $b^{2}$ vorliegt, obwohl die Anfangsbreite schon abgezogen wurde. Diese Verbreiterung muß also nach der Anregung erfolgt sein. Zunächst würde man vermuten, daß sie durch Dickeninhomogenitäten bedingt sein könnte. Trägt man jedoch diesen stets gefundenen Achsenabschnitt $b$ gegen die optisch gemessene Dickeninhomogenität für verschiedene Kristalle auf, so findet man durch Extrapolation, daß auch bei ideal planparallelen Kristallen noch ein Rest $b_{0}$ bleibt (s. Abb. 10). Es muß sich

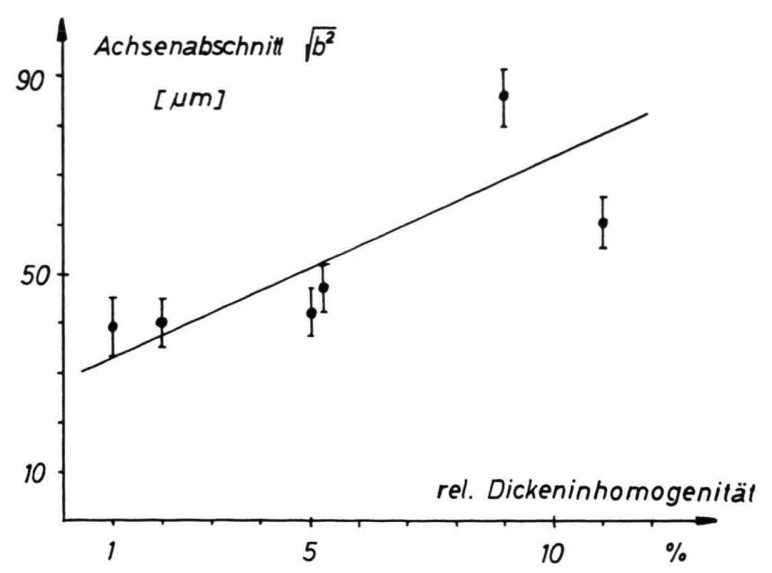

Abb. 10. Achsenabschnitte $b$ nach Abb. 9 aufgetragen für verschiedene Kristalle gegen deren jeweilige relative Dickeninhomogenität.

also um eine echte, laufzeitunabhängige Verbreiterung der Verteilung handeln, die beim Durchqueren des Kristalls hinzukommt. Sie kann bis jetzt nicht eindeutig erklärt werden.

Zieht man diese konstante Verbreiterung von den gemessenen Werten ab und trägt man die so korri- gierten Werte logarithmisch gegen die Transitzeit auf, so kann man prüfen, welchem Zeitgesetz die Verbreiterung gehorcht, s. Abb. 1l. Für höhere Lichtintensitäten folgt das lineare Zeitgesetz der Coulomb-Verbreiterung, für kleine Lichtintensitäten in guter Näherung das Wurzelgesetz der Diffusion, das schon durch die Gerade der Abb. 9 bestätigt wurde. In Ergänzung zu den Abschätzungen der Tab. 1 ist durch Vergleich mit der 100\%-Kurve der Abb. 11 auch experimentell sichergestellt, daß bei den schwachen Lichtintensitäten kein wesentlicher Anteil Coulomb-Verbreiterung mitgemessen wurde.

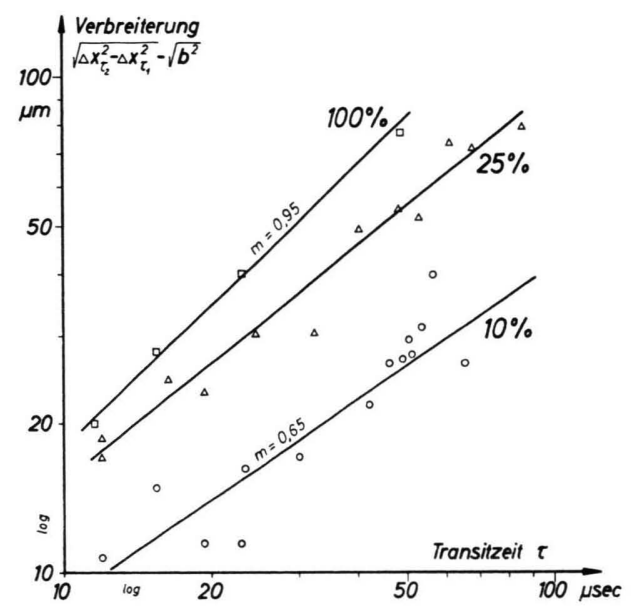

Abb. 11. Zeitabhängigkeit der Verbreiterung bei verschiedenen Anregungsintensitäten: bei höheren Intensitäten überwiegt die Coulomb-Verbreiterung (Steigung 1, d. h. $\sim t$ ) über die Diffusions-Verbreiterung (Steigung $\frac{1}{2}$, d. h. $\sim \sqrt{t}$ ).

\section{Zusammenfassung und Diskussion der Ergebnissse}

Im theoretischen Teil der vorliegenden Arbeit wurde gezeigt, daß die Driftstromimpulse nach KePler und LeBlanc ${ }^{1}$ nach Differentiation im wesentlichen die Verteilungsfunktion der driftenden Raumladung ergeben, wenn deren Breite klein gegen die Kristalldicke ist. Damit ergibt sich eine Möglichkeit, Diffusionsverbreiterungen experimentell zu messen. Da im Gegensatz zu den klassischen Halbleitern hier echte Überschußraumladungen vorliegen, ist die Verbreiterung durch Coulomb-Wechselwirkung unter den Ladungsträgern nur durch sehr geringe Anregung klein zu halten, wodurch die Impulsströme so schwach werden, daß man nahe an die Grenze der Meßbarkeit gelangt. Bei sehr guten Anthracenkristallen gelingt es jedoch, eine Reihe 
weiterer Störungen weitgehend auszuschalten und tatsächlich die Diffusionsverbreiterung quantitativ zu erfassen. Die Messungen ergeben in $a$-Richtung eine Elektronen-Diffusionskonstante von $D=0,06$ $\mathrm{cm}^{2} / \mathrm{sec}$, die im Rahmen der Meßgenauigkeit mit dem aus der Beweglichkeit errechneten Wert von $D=0,044 \mathrm{~cm}^{2} / \mathrm{sec}$ übereinstimmt.

Da flache Haftstellen die Beweglichkeit verringern, die Diffusionskonstante bei der angegebenen Meßtechnik jedoch (scheinbar) vergrößern, kann die Übereinstimmung auch als Beweis dafür angesehen werden, daß die Elektronen wirklich frei sind.

Die Ergebnisse könnten von der Meßtechnik her noch in folgender Weise verbessert werden:
1. Mittelung mehrerer Messungen in einem Vielkanalanalysator.

2. Messungen an dünneren Kristallen mit größerer Fläche (wobei die Dicke jedoch groß gegen die Eindringtiefe des Anregungslichts bleiben muß).

3. Optischer Nachweis des Ankommens der Ladungsträger an der Gegenelektrode durch Rekombinationslumineszenz in einer geeigneten Aufdampfschicht.

Die Autoren Karl und Seeger danken Herrn Prof. Dr. F. Matossi, die Autoren KarL und Schmid Herrn Prof. Dr. H. C. Wolf für die freundliche und wohlwollende Unterstützung der Arbeiten. Die Fraunhofergesellschaft München und die Deutsche Forschungsgemeinschaft haben wertwolle finanzielle Förderung geleistet. Herr Dipl.-Phys. K. W. BENZ führte dankenswerterweise die Messungen der Lebensdauer der TriplettExcitonen durch.

\title{
Brillouin-Streuung in Strontiumtitanat-Einkristallen im Temperaturbereich $5{ }^{\circ} \mathrm{K}$ bis $300^{\circ} \mathrm{K}$
}

\author{
A. Laubereau und R. ZureK * \\ Physik-Department der Technischen Hochschule München \\ (Z. Naturforsch. 25 a, 391-401 [1970]; eingegangen am 18. Dezember 1969)
}

\begin{abstract}
Brillouin-Streuexperimente in $\mathrm{SrTiO}_{3}$ im Bereich der Phasenumwandlung von der kubischen zur tetragonalen Struktur werden mit Hilfe eines Modells für den Phasenübergang gedeutet, das Ergebnisse liefert, die der Theorie von Landau und Khalatnikow entsprechen. Die Phasenänderung wird als Übergang 2. Ordnung betrachtet, bei dem die beobachteten akustischen Effekte unterhalb der Umwandlungstemperatur auftreten. Als Temperatur des Phasenüberganges wird der Wert $107 \pm 0,5^{\circ} \mathrm{K}$ ermittelt. Die Größe der Stufe in den Brillouin-Verschiebungen konnte aus Literaturdaten, wie Änderungen der Gitterparameter, Drehwinkel der Sauerstoffoktaeder, soft-mode-Frequenzen, vorhergesagt werden. Die Ủbereinstimmung mit den experimentellen Ergebnissen ist zufriedenstellend. Die Messungen legen eine verhältnismäßig geordnete Domänenstruktur der untersuchten Kristallproben in der Tieftemperaturphase nahe mit einer Domänenorientierung entlang den Achsenrichtungen der kubischen Phase und überwiegend antiparallel ausgerichteten tetragonalen Domänenachsen. Die beim Anwenden einer äußeren Spannung im Brillouin-Spektrum beobachteten Änderungen werden als Umorientierung der Domänenachsen gedeutet.
\end{abstract}

\section{A. Einleitung}

Strontiumtitanat weist einen nicht ferroelektrischen Phasenübergang bei etwa $110^{\circ} \mathrm{K}$ auf. Von der kubischen Perowskitstruktur oberhalb der Um-

Sonderdruckanforderungen an A. Laubereau, PhysikDepartment der Technischen Hochschule, D-8000 München 2, Arcisstraße 21.

* Auszug aus der von der Fakultät für Allgemeine Wissenschaften der Technischen Hochschule genehmigten Dissertation über „Die Untersuchung der Brillouin-Streuung in Strontiumtitanat-Einkristallen im Temperaturbereich $5^{\circ} \mathrm{K}$ bis $300^{\circ} \mathrm{K}$ “ des Dipl.-Ing. Rudolf Zurek. wandlungstemperatur erfolgt ein Übergang zur tetragonalen Struktur ${ }^{1,2}$. Während sich die Gitterparameter in der Nachbarschaft der Umwandlungstemperatur nur sehr wenig ändern ${ }^{3,4}$, sind mit dem Phasenübergang auffällige Änderungen in den aku-

1 K. A. Müller, Helv. Phys. Acta 31, 173 [1958] ; Phys. Rev. Letters 2, 341 [1959].

2 L. Rimai u. G. de Mars, Phys. Rev. 127, 709 [1962].

3 F. W. Lytle, J. Appl. Phys. 35, 2212 [1964].

4 B. Alefeld, Z. Physik 222, 155 [1969]. 